\section{Informed consent: cultural differences}

Efforts to improve informedconsent forms for clinical trials should not overlook difficulties faced by patients in non-Western settings (Nature 482, 16; 2012).

Institutions in developed countries are expanding clinical trials in Africa and Asia, and most focus on the signing of the consent form rather than on the exchange of information between researchers and potential participants.

Information should be culturally adjusted, taking local factors into account. These might include degrees of illiteracy, native dialects of ethnic minorities, a lack of suitable vocabulary, a preference for communal decision-making, and stigmatization by local authorities if people do not sign.

Alternative ways of obtaining consent could be used more often, including witnessing oral consent, verifying the quality of information for participants, or using videos to present the study and record consent.

Revising consent forms could help to reassess the concept of globalized bioethics, preventing controversial practices such as those used in researching the genetics of the Havasupai tribe (J. Couzin-Frankel Science 328, 558; 2010).

Gilles Guerrier, Didier Sicard, Paul T. Brey Institut Pasteur du Laos, Vientiane, Laos. guerriergilles@gmail.com

\section{Informed consent: meet patients' needs}

We agree that informed-consent forms should be improved (Nature 482, 16; 2012) beyond shortening and simplifying them. Participants need to know whether a trial is addressing an unmet clinical need, and that the test is necessary to answer a key question.

For example, a 'me-too' drug that seeks marketing authorization by comparison against a placebo, rather than against alternative products, will not solve a patient's unmet need. Neither is it in participants' best interests for a trial to establish the acceptability of a drug with inferior efficacy that provides no other advantage. If the informedconsent form were to explain these points, it is likely that far fewer patients would agree to participate.

Patients also need to understand why randomization is the only scientific and ethical way to provide meaningful results when a trial's outcome is unpredictable. Any other strategy, such as giving or withholding an untested drug, would be arbitrary and would not clarify the uncertainty.

Details of the therapeutic rationale, and of the pharmacological and toxicological properties of the experimental intervention, should be provided to trial participants on request.

Informed consent should reassure patients that trials aim to help them, and will allow onto the market only products that do so, rather than products that just meet commercial expectations. There is a long way to go in cleaning up clinical trials before the trust of patients is regained. Silvio Garattini, Vittorio Bertele', Rita Banzi Mario Negri Institute for Pharmacological Research, Milan, Italy. vittorio.bertele@marionegri.it

\section{Citations: not all measures are equal}

The scientific community needs to be aware of the limitations of Google Scholar's personalized citation reports. Clicking on 'My citations' on the site may offer a nice ego boost, but I would not recommend using the reports for decisions that could affect careers.

Google Scholar overestimates the number of citable articles (in comparison with formal citation services such as Scopus and
Thomson Reuters) because of the automated way it collects data, including 'grey' literature such as theses. For my own publications, for example, Google Scholar yields $38 \%$ more citations and boosts the $h$-index by $26 \%$.

A citation report for one of my articles revealed that Google Scholar had counted as independent citations four web pages on which authors had posted copies of their articles, plus one listing only an article title; and one to a paper in which my name didn't appear. Personalized searches by my colleagues exposed comparable errors.

These drawbacks might also allow unscrupulous individuals to use such tactics to inflate their citation reports, particularly as independent vetting is blocked by password access.

Scott L. Hooper Ohio University, Athens, USA.hooper@ohio.edu

\section{Citations: results differ by database}

Databases such as Thomson Reuters' ISI Web of Science, Scopus, Google Scholar and Microsoft's Academic Search allow authors to compute their own citation statistics, but they yield inconsistent results.

The discrepancies come from differences in information sources and in temporal citation coverage. Web of Science and Scopus, for example, provide citation data only for their indexed journals, giving different coverage for the number of journals, precursor articles and fields of academic research often with regional biases (such as European versus US sources). Google Scholar includes all journals (indexed, free access and popular science), conference proceedings, books, theses, reports, local press and electronic sources - all subject to variable degrees of control and scrutiny.

A debate is crucial on how these tracking tools compare and should be used, given that their indiscriminate usage has potentially negative implications for academic careers.

Carles Alcaraz, Sofia Morais Institute of Agrifood Research and Technology (IRTA), Sant Carles de la Ràpita, Spain. carles.alcaraz@gmail.com

\section{Track illegal trade in wildlife}

Illegal wildlife trade is the secondlargest black market worldwide, after narcotics. More effective strategies must be devised to intercept the first links of the wildlife-trade chain and beyond.

A lack of resources in tropical countries often undermines existing legal frameworks for preventing wildlife trading. Local governments pay scant attention to the trade because it is not perceived as a major threat to biodiversity or to human well-being. A large volume of wildlife trade is international increasing the risk of biological invasions and of spreading zoonotic diseases.

National environmental agencies should collaborate to centralize the collection and organization of local data to feed into international wildlifetrade databases such as the Convention on International Trade in Endangered Species of Wild Fauna and Flora (CITES). This would help to identify the species that are most threatened by trade, determine major harvesting sites and routes, and locate sources of demand and supply - as well as revealing the extent of enforcement and of currently unreported shipments. Governments from developing and developed nations could then weigh in with policy improvements.

Luís Felipe Toledo State University of Campinas, São Paulo, Brazil. toledolf2@yahoo.com Marianne V. Asmüssen, Jon Paul Rodríguez Venezuelan Institute for Scientific Research (IVIC), Venezuela; and EcoHealth Alliance, New York, USA. 